\title{
The Invariant Charges of the Nambu-Goto Theory in WKB-Approximation: Renormalization`
}

\author{
K. Pohlmeyer
}

Fakultät für Physik der Universität Freiburg, D-7800 Freiburg, Federal Republic of Germany

\begin{abstract}
We discuss the one loop renormalization of the reparametrization invariant non-local conserved charges of the Nambu-Goto string theory. In addition we show the stability of a special well-known state under the corresponding infinitesimal symmetry transformations - at least in the WKBapproximation.
\end{abstract}

\section{Classical Considerations}

The classical Nambu-Goto string theory possesses infinitely many independent, reparametrization invariant non-local conserved charges [1] which act as infinitesimal generators of symmetry transformations. Here we want to argue in the Euclidean version that these charges can be carried over to the quantum theory as well-defined operators - at least in WKB-approximation - without introducing unfamiliar counterterms. Further, we want to demonstrate that the renormalized loop wave-functional $\psi(\mathscr{C})$ constructed by the authors of [2] is invariant under the above symmetry transformations - at least in WKB-approximation. Thus, $\psi(\mathscr{C})$ is likely to correspond to the "Euclidean" ground state of the system.

Associated with bosonic Euclidean closed strings are closed curves $\mathscr{C}$ in $\mathbb{R}^{d}$, $d=3,4, \ldots$. Let $x_{\mu}=x_{\mu}(\sigma)=x_{\mu}(\sigma+2 \pi), \sigma \in \mathbb{R}$ be a parametrization of $\mathscr{C}$ and let $M$ be a constant mass parameter. Classically the invariant charges in question are given in terms of cyclic sums of path-ordered multiple integrals:

$$
\begin{aligned}
\mathscr{Z}_{\mu_{1} \ldots \mu_{N}}^{ \pm}= & \int_{0}^{2 \pi} d \sigma_{1} \ldots \int_{0}^{2 \pi} d \sigma_{N} \theta\left(\sigma_{1}-\sigma_{2}\right) \ldots \theta\left(\sigma_{N-1}-\sigma_{N}\right) \\
& \times\left[\frac{1}{i} \frac{\delta}{\delta x_{\mu_{1}}\left(\sigma_{1}\right)} \pm M^{2} x_{\mu_{1}}^{\prime}\left(\sigma_{1}\right)\right] \ldots\left[\frac{1}{i} \frac{\delta}{\delta x_{\mu_{N}}\left(\sigma_{N}\right)} \pm M^{2} x_{\mu_{N}}^{\prime}\left(\sigma_{N}\right)\right] \\
& + \text { cyclic permutations of the indices } \mu_{1}, \ldots, \mu_{N} .
\end{aligned}
$$

\footnotetext{
* Work supported by Deutsche Forschungsgemeinschaft •
} 
Here $\theta(\sigma)$ denotes the step function

$$
\theta(\sigma)=\left\{\begin{array}{lll}
1 & \text { for } & \sigma \geqq 0 \\
0 & \text { for } & \sigma<0
\end{array}\right.
$$

The dash denotes differentiation with respect to the parameter $\sigma: x_{\mu}^{\prime}(\sigma)=\frac{d}{d \sigma} x_{\mu}(\sigma)$. Planck's constant $\hbar$ has been absorbed in the mass parameter $M$. The action of the operators $\left[\frac{1}{i} \frac{\delta}{\delta x_{\mu_{j}}\left(\sigma_{j}\right)} \pm M^{2} x_{\mu_{j}}^{\prime}\left(\sigma_{j}\right)\right]$ is restricted: each one of these operators acts only on the leading term in the $1 / M^{2}$-expansion of the wave-functional of the state to which the charge $\mathscr{Z}_{\mu_{1} \ldots \mu_{N}}^{ \pm}$is applied.

In [2] a particular well-defined, reparametrization invariant wave-functional $\psi(\mathscr{C})$ was derived which solves in the leading and the first non-leading order in $1 / M^{2}$ (i.e. in $\hbar$ ) the renormalized loop equation. It turned out necessary to add a counterterm involving the curvature of the "trajectory" surface spanned by the curve $\mathscr{C}$ to the original action given just by its area. As a consequence, there appears a new dimensionless coupling constant $\gamma$.

For closed, infinite differentiable and double point free curves $\mathscr{C}$ the wavefunctional $\psi(\mathscr{C})$ in WKB-approximation is given by

$$
\psi(\mathscr{C})=\text { Const } M e^{-M^{2} A(\mathscr{C})} \exp \left\{\gamma \kappa(\mathscr{C})-\frac{1}{2} W_{P V}\left(\Delta^{T}\right)\right\} .
$$

Here the symbol $A(\mathscr{C})$ denotes the Euclidean area of the minimal surface $\Sigma$ enclosed by the curve $\mathscr{C}, \Sigma$ being parametrized by $\Gamma \rightarrow \mathbb{R}^{d}: z \rightarrow \varphi_{\mu}(z)$.

$$
\begin{gathered}
A(\mathscr{C})=\int_{\Gamma} d^{2} z|g(z)|^{1 / 2}, \\
|g(z)| \doteq \operatorname{det} g_{a b}(z), \quad g_{a b}(z) \doteq\left(\partial_{a} \varphi\right)(z) \cdot\left(\partial_{b} \varphi\right)(z), \quad \partial_{a} \doteq \frac{\partial}{\partial z^{a}}, \\
g_{a b} g^{b c} \doteq \delta_{a}^{c}, \quad-|g|^{-1 / 2} \partial_{a}|g|^{1 / 2} g^{a b} \partial_{b} \varphi_{\mu}=0 .
\end{gathered}
$$

The symbol $\kappa(\mathscr{C})$ stands for the integral

$$
\kappa(\mathscr{C})=2 \int_{\Gamma} d^{2} z|g(z)|^{1 / 2} K(z),
$$

where $K$ denotes the Gaussian curvature of the surface $\Sigma: K(z) \leqq 0 . \gamma$ is a free real dimensionless parameter.

The symbol $W_{P V}\left(\Delta^{T}\right)$ signifies the finite part of the logarithm of the determinant of the transversal fluctuation operator $\Delta^{T}$

$$
W_{P V}\left(\Delta^{T}\right)=-\alpha_{0} C-\int_{0}^{\infty} \frac{d t}{t}\left\{\operatorname{Tr} e^{-t H}-\alpha_{1} t^{-1}-\alpha_{1 / 2} t^{-1 / 2}-\alpha_{0} \theta(1-t)\right\}
$$

where the Schwarz operator $H$ is defined by

$$
H=-|g|^{-1 / 2} D_{a}|g|^{1 / 2} g^{a b} D_{b}+U
$$

with homogeneous Dirichlet boundary conditions on $\partial \Gamma$. Here $D_{a}$ denotes the covariant derivative

$$
D_{a}^{i j} \doteq \partial_{a} \delta^{i j}+A_{a}^{i j}, \quad A_{a}^{i j}=\left(e^{i} \cdot \partial_{a} e^{j}\right), \quad i, j=1, \ldots, d-2,
$$


and $U$ is given in terms of the second fundamental form of the surface $\Sigma: V_{a b}^{i}(z)$ $=-\left(\partial_{a} e^{i} \cdot \partial_{b} \varphi\right)(z), i=1, \ldots, d-2$ by

$$
\begin{gathered}
U^{i j}=|g|^{-1} \varepsilon_{a c} \varepsilon_{b d} V_{a b}^{i} V_{c d}^{j}, \\
\varepsilon_{a b} \doteq\left\{\begin{aligned}
+1 & \text { for } a=1, b=2 \\
-1 & \text { for } a=2, b=1 \\
0 & \text { otherwise. }
\end{aligned}\right.
\end{gathered}
$$

The vectors $e_{\mu}^{i}=e_{\mu}^{i}(z), i=1, \ldots, d-2$ provide an orthonormal basis of the complement of the tangent space to $\Sigma$ at $\varphi(z) . \alpha_{0}, \alpha_{1 / 2}$, and $\alpha_{1}$ are Seeley coefficients of $\Delta^{T}$, alias $H[2], C$ is Euler's constant.

We want to show that the state $\psi(\mathscr{C})$ to the leading and next to leading order in $1 / M^{2}$ is left invariant under the symmetry transformations generated by the charges $\mathscr{Z}_{\mu_{1} \ldots \mu_{N}}^{ \pm}, 1 \leqq \mu_{j} \leqq d ; j=1, \ldots, N ; N=1,2, \ldots$. In the leading order in $1 / M^{2}$

$$
\begin{aligned}
\mathscr{Z}_{\mu_{1} \ldots \mu_{N}}^{ \pm} \psi(\mathscr{C})= & {\left[M^{2}\right]^{N}\left\{\int_{0}^{2 \pi} d \sigma_{1} \int_{0}^{\sigma_{1}} d \sigma_{2} \ldots \int_{0}^{\sigma_{N}-1} d \sigma_{N}\left[i p_{\mu_{1}}\left(\sigma_{1}\right) \pm x_{\mu_{1}}^{\prime}\left(\sigma_{1}\right)\right] \ldots\right.} \\
& \left.\ldots\left[i p_{\mu_{N}}\left(\sigma_{N}\right) \pm x_{\mu_{N}}^{\prime}\left(\sigma_{N}\right)\right]+\operatorname{cycl}\right\} \psi(\mathscr{C}),
\end{aligned}
$$

where

$$
\begin{aligned}
& p_{\mu}(\sigma) \doteq \frac{\delta A(\mathscr{C})}{\delta x_{\mu}(\sigma)}=\left|x^{\prime}(\sigma)\right| n^{a}(\sigma)\left(\partial_{a} \varphi_{\mu}\right)(z(\sigma)), \\
& n^{a}(\sigma) \doteq|g(z: g(z(\sigma))(\sigma))|^{1 / 2} g^{a b}(z(\sigma)) \varepsilon_{b c} \dot{z}^{c}(\sigma) .
\end{aligned}
$$

The dot stands for differentiation with respect to the arc length: $\frac{d}{d s} \cdot z=z(\sigma)$ $=z(\sigma+2 \pi)$ is a parametrization of the boundary $\partial \Gamma$ of $\Gamma$.

The minimal surface $\Sigma$ of area $A$ is mapped onto its conjugate minimal surface $\tilde{\Sigma}$ of the same area $A$ defined up to a global translation with the help of the parametrization $\Gamma \rightarrow \mathbb{R}^{d}: z \rightarrow \psi_{\mu}(z)$, where

$$
\left(\partial_{a} \psi_{\mu}\right)(z)=-\varepsilon_{a b}|g(z)|^{1 / 2} g^{b c}(z)\left(\partial_{c} \varphi_{\mu}\right)(z) .
$$

A point on the boundary curve $\mathscr{C}$ of $\Sigma: x_{\mu}(\sigma)=\varphi_{\mu}(z(\sigma))$ with unit tangent vector $\dot{x}_{\mu}(\sigma)$ and unit outward normal vector in the surface $\Sigma: p_{\mu}(\sigma) /\left|x^{\prime}(\sigma)\right|$ is mapped to a point on the boundary curve $\widetilde{\mathscr{C}}$ of $\tilde{\Sigma}: \psi_{\mu}(z(\sigma))$ with unit tangent vector $p_{\mu}(\sigma) /\left|x^{\prime}(\sigma)\right|$ and unit outward normal vector in the surface $\tilde{\Sigma}:-\dot{x}_{\mu}(\sigma)$. The curves $\mathscr{C}$ and $\widetilde{\mathscr{C}}$ have equal lengths (cf., for instance, [3, Sect. 121]). We set

$$
\chi_{\mu}^{ \pm}(z)=i \psi_{\mu}(z) \pm \varphi_{\mu}(z)
$$

and define for $2 \pi>\sigma \geqq \lambda \geqq 0$ the path-ordered integrals

$$
\begin{aligned}
R_{\mu_{1} \ldots \mu_{N}}^{ \pm \psi}(z(\sigma), z(\lambda))= & \int_{\lambda}^{\sigma} d \sigma_{1} \int_{\lambda}^{\sigma_{1}} d \sigma_{2} \ldots \int_{\lambda}^{\sigma_{N}-1} d \sigma_{N}\left[i p_{\mu_{1}}\left(\sigma_{1}\right) \pm x_{\mu_{1}}^{\prime}\left(\sigma_{1}\right)\right] \ldots \\
& \ldots\left[i p_{\mu_{N}}\left(\sigma_{N}\right) \pm x_{\mu_{N}}^{\prime}\left(\sigma_{N}\right)\right] \\
& =\int_{\lambda}^{\sigma} d \sigma_{1} \int_{\lambda}^{\sigma_{1}} d \sigma_{2} \ldots \int_{\lambda}^{\sigma_{N}-1} d \sigma_{N} \chi_{\mu_{1}}^{ \pm \prime}\left(z\left(\sigma_{1}\right)\right) \ldots \chi_{\mu_{N}}^{ \pm \prime}\left(z\left(\sigma_{N}\right)\right)
\end{aligned}
$$


The translation invariance of the state $\psi(\mathscr{C})$ implies

$$
P_{\mu_{1}}^{\psi}=\lim _{\sigma \uparrow 2 \pi} R_{\mu_{1}}^{ \pm \psi}(z(\sigma), z(0))=0 .
$$

We shall have proved in leading order in $1 / M^{2}$ that $\psi(\mathscr{C})$ is annihilated by the invariant charges $\mathscr{Z}_{\mu_{1} \ldots \mu_{N}}^{ \pm}$if we can show that all multiple integrals $R_{\mu_{1} \ldots \mu_{N}}^{ \pm \psi}(z(\sigma), z(0))$ vanish once the variable $\sigma$ takes the value $2 \pi$.

More generally, we shall prove that $R_{\mu_{1} \ldots \mu_{N}}^{ \pm \psi}(z(\sigma), z(0))$ defined on the boundary curve of $\Gamma$ can be continued to single-valued functions $R_{\mu_{1} \ldots \mu_{N}}^{ \pm \psi}(z, z(0))$ over $\Gamma$.

Proof by Induction. The claim is true for $N=1: \chi_{\mu_{1}}^{ \pm}(z)=R_{\mu_{1}}^{ \pm \psi}(z, z(0))$. Assume that all path-ordered integrals $R_{\mu_{1} \ldots \mu_{n}}^{ \pm \psi}(z(\sigma), z(0))$ with $n<N(N \geqq 2)$ can be continued to single-valued functions $R_{\mu_{1} \ldots \mu_{n}}^{ \pm \psi}(z, z(0))$ over the interior of $\Gamma$, in particular, that

$$
\lim _{\sigma \uparrow 2 \pi} R_{\mu_{1} \ldots \mu_{n}}^{ \pm \psi}(z(\sigma), z(0))=0 .
$$

Then for any closed curve $z=\zeta(\sigma), 0 \leqq \sigma \leqq 2 \pi$ contained in $\Gamma$-its interior will be denoted by $\Gamma^{\prime}-$

$$
\begin{aligned}
& \int_{0}^{2 \pi} d \sigma \chi_{\mu_{1}}^{ \pm \prime}(\zeta(\sigma)) R_{\mu_{2} \ldots \mu_{N}}^{ \pm \psi}(\zeta(\sigma), z(0))= \pm i \int_{0}^{2 \pi} d \sigma\left(\partial_{n} \chi_{\mu_{1}}^{ \pm}\right)(\zeta(\sigma)) R_{\mu_{2} \ldots \mu_{N}}^{ \pm \psi}(\zeta(\sigma), z(0)) \\
& = \pm i \int_{\Gamma^{\prime}} d^{2} z|g(z)|^{1 / 2}\left(\partial_{a} \chi_{\mu_{1}}^{ \pm}\right)(z) g^{a b}(z)\left(\partial_{b} \chi_{\mu_{2}}^{ \pm}\right)(z) R_{\mu_{3} \ldots \mu_{N}}^{ \pm \psi}(z, z(0))=0
\end{aligned}
$$

because of the identity $\left(\partial_{a} \chi_{\mu}^{ \pm}\right)(z) g^{a b}(z)\left(\partial_{b} \chi_{v}^{ \pm}\right)(z)=0$. This means that also the integrals

$$
R_{\mu_{1} \ldots \mu_{N}}^{ \pm \psi}(z(\sigma), z(0))=\int_{0}^{\sigma} d \sigma_{1} \chi_{\mu_{1}}^{ \pm \prime}\left(z\left(\sigma_{1}\right)\right) R_{\mu_{2} \ldots \mu_{N}}^{ \pm \psi}\left(z\left(\sigma_{1}\right), z(0)\right)
$$

with $z=z(\sigma) 0 \leqq \sigma<2 \pi$ parametrizing the boundary curve $\partial \Gamma$ can be continued to single-valued functions $R_{\mu_{1} \ldots \mu_{N}}^{ \pm \psi}(z, z(0))$ over $\Gamma$ and that in particular $\lim _{\sigma \uparrow 2 \pi} R_{\mu_{1} \ldots \mu_{N}}^{ \pm \psi}(z(\sigma), z(0))$ vanish. Thus the induction hypothesis is also valid for $n=N$.

This completes the proof that to leading order in $1 / M^{2}, \psi(\mathscr{C})$ is annihilated by the invariant charges $\mathscr{Z}_{\mu_{1} \ldots \mu_{N}}^{ \pm}$.

By a similar argument to the one just given we conclude that for $1 \leqq \mu_{j} \leqq d$; $j=1, \ldots, N ; N=1,2, \ldots$,

$$
\lim _{\sigma \uparrow 2 \pi} R_{\mu_{1} \ldots \mu_{N}}^{ \pm \psi}(z(\sigma), z(\lambda)), \quad 2 \pi>\lambda \geqq 0
$$

can be continued to single-valued functions $\lim _{\sigma \uparrow 2 \pi} R_{\mu_{1} \ldots \mu_{N}}^{ \pm \psi}(z(\sigma), z)$ over $\Gamma$. Actually,

$$
\lim _{\sigma \uparrow 2 \pi} R_{\mu_{1} \ldots \mu_{N}}^{ \pm \psi}(z(\sigma), z)=(-1)^{N} R_{\mu_{N} \ldots \mu_{1}}^{ \pm \psi}(z, z(0))
$$

Moreover, for infinite differentiable and double point free curves $\mathscr{C}$ the pathordered integrals $R_{\mu_{1} \ldots \mu_{N}}^{ \pm \psi}(z(\sigma), z(0))$ are infinite differentiable complex periodic functions in $\sigma$ of period $2 \pi$ with an $N$-fold zero at $\sigma=0,2 \pi, \ldots$. Also, $R_{\mu_{1} \ldots \mu_{N}}^{ \pm \psi}(z(\sigma), z(\lambda))$ are infinite differentiable complex functions of $\sigma$ and $\lambda$ periodic of period $2 \pi$ in both variables separately with $N$-fold zeros at $\sigma=\lambda$ modulo $2 \pi$. 
This information will be useful in the sequel when we study the action of the invariant charges $\mathscr{Z}_{\mu_{1} \ldots \mu_{N}}^{ \pm}$or rather their building blocks on the state $\psi(\mathscr{C})$ in the next to leading order in the $1 / M^{2}$ expansion.

\section{The Invariant Charges in WKB-Approximation}

We are faced with the problem to turn the formal expressions for the invariant charges in the Schrödinger representation

$$
\begin{aligned}
\mathscr{Z}_{\mu_{1} \ldots \mu_{N}}^{ \pm}= & {\left[M^{2}\right]^{N} \oint d \sigma_{N} \int_{\sigma_{N}}^{2 \pi+\sigma_{N}} d \sigma_{1} \ldots \int_{\sigma_{N}}^{2 \pi+\sigma_{N}} d \sigma_{N-1} \theta\left(\sigma_{1}-\sigma_{2}\right) \ldots \theta\left(\sigma_{N-2}-\sigma_{N-1}\right) } \\
& \times \hat{u}_{\mu_{1}}^{ \pm}\left(\sigma_{1}\right) \ldots \hat{u}_{\mu_{N}}^{ \pm}\left(\sigma_{N}\right)
\end{aligned}
$$

with

$$
\hat{u}_{\mu}^{ \pm}(\sigma)=\frac{1}{M^{2}} \frac{\delta}{i \delta x_{\mu}(\sigma)} \pm x_{\mu}^{\prime}(\sigma)
$$

(cf. [4]) into well-defined operators in WKB-approximation. This approximation arises from the classical one by slightly relaxing the restriction on the action of the operators $\hat{u}_{\mu_{j}}^{ \pm}\left(\sigma_{j}\right)$ : all of these operators with one possible exception act only on the leading term in the $1 / M^{2}$-expansion of the wave-functional of the state to which $\mathscr{Z}_{\mu_{1} \ldots \mu_{N}}^{ \pm}$has been applied. Each operator $\hat{u}_{\mu_{j}}^{ \pm}\left(\sigma_{j}\right)$ in its turn plays the part of the exceptional one. As such it may either act on the next to leading term in the $1 / M^{2}$-expansion of the wave-functional or on the factors in front of the wavefunctional resulting from the "classical" action of the operators standing on its right. In the latter case it must be assumed that the order of the operators has been specified and that appropriate counterterms have been added.

Only those definitions of $\mathscr{Z}_{\mu_{1} \ldots \mu_{N}}^{ \pm}$in terms of products of $\hat{u}_{\mu_{j}}^{ \pm}\left(\sigma_{j}\right)$ operators are admitted which preserve the independence of the initial and final point of integration and the invariance of $\mathscr{Z}_{\mu_{1} \ldots \mu_{N}}^{ \pm}$under cyclic permutations of the tensor indices. More generally, the passage to the quantum theory should be such that all linear identities continue to hold. Two particular orderings suggest themselves: cyclic path-ordering:

$$
\begin{aligned}
P \hat{u}_{\mu_{1}}^{ \pm}\left(\sigma_{1}\right) \ldots \hat{u}_{\mu_{N}}^{ \pm}\left(\sigma_{N}\right)= & \frac{1}{N} \sum_{j=1}^{N} \hat{u}_{\mu_{\pi(j)}}^{ \pm}\left(\sigma_{\pi(j)}\right) \hat{u}_{\mu_{\pi(j+1)}}^{ \pm}\left(\sigma_{\pi(j+1)}\right) \ldots \hat{u}_{\mu_{\pi(N)}}^{ \pm}\left(\sigma_{\pi(N)}\right) \\
& \times \hat{u}_{\mu_{\pi(1)}}^{ \pm}\left(\sigma_{\pi(1)}\right) \ldots \hat{u}_{\mu_{\pi(j-2)}}^{ \pm}\left(\sigma_{\pi(j-2)}\right) \hat{u}_{\mu_{\pi(j-1)}}^{ \pm}\left(\sigma_{\pi(j-1)}\right)
\end{aligned}
$$

and cyclic anti path-ordering:

$$
\begin{aligned}
\bar{P} \hat{u}_{\mu_{1}}^{ \pm}\left(\sigma_{1}\right) \ldots \hat{u}_{\mu_{N}}^{ \pm}\left(\sigma_{N}\right)= & \frac{1}{N} \sum_{j=1}^{N} \hat{u}_{\mu_{\pi(j)}}^{ \pm}\left(\sigma_{\pi(j)}\right) \hat{u}_{\mu_{\pi(j-1)}}^{ \pm}\left(\sigma_{\pi(j-1)}\right) \ldots \hat{u}_{\mu_{\pi(1)}}^{ \pm}\left(\sigma_{\pi(1)}\right) \\
& \times \hat{u}_{\mu_{\pi(N)}^{ \pm}}^{ \pm}\left(\sigma_{\pi(N)}\right) \ldots \hat{u}_{\mu_{\pi(j+2)}}^{ \pm}\left(\sigma_{\pi(j+2)}\right) \hat{u}_{\mu_{\pi(j+1)}^{ \pm}}^{ \pm}\left(\sigma_{\pi(j+1)}\right)
\end{aligned}
$$

Here $x_{\mu}=x_{\mu}(\sigma): x_{\mu}(\sigma+2 \pi)=x_{\mu}(\sigma)$ is a parametrization of the curve $\mathscr{C}$ such that the curve $\mathscr{C}$ oriented in the direction of increasing values of $\sigma$ surrounds the 
minimal surface $\Sigma$ counter-clockwise and the permutation $\pi$

$$
\left(\begin{array}{cccc}
1 & 2 & \ldots & N \\
\pi(1) & \pi(2) & \ldots & \pi(N)
\end{array}\right)
$$

- determined up to an element of the cyclic group - is such that

$$
\sigma_{\pi(1)}>\sigma_{\pi(2)}>\ldots>\sigma_{\pi(N)},
$$

turning once around the minimal surface counter-clockwise. Since neither ordering is preferred to the alternative one, we are led to take the average

$$
\frac{1}{2}(P+\bar{P}) \hat{u}_{\mu_{1}}^{ \pm}\left(\sigma_{1}\right) \ldots \hat{u}_{\mu_{N}}^{ \pm}\left(\sigma_{N}\right)
$$

For the WKB-approximation this is equivalent to total symmetrization of operator products.

Thus we arrive at the still formal expression for the invariant charges:

$$
\begin{aligned}
& \mathscr{Z}_{\mu_{1} \ldots \mu_{N}}^{ \pm}=\mathscr{Z}_{\mu_{1} \ldots \mu_{N}}^{ \pm c l}+\left[M^{2}\right]^{N-1} \\
& \times \sum_{j=1}^{N} \oint d \sigma_{j} \hat{R}_{\mu_{j+1} \ldots \mu_{N} \ldots \mu_{j-1}}^{ \pm c l}\left(2 \pi+\sigma_{j}, \sigma_{j}\right)\left(\frac{\delta}{i \delta x_{\mu_{j}}\left(\sigma_{j}\right)}\right)_{\mathrm{WKB}} \\
& +\frac{1}{2}\left[M^{2}\right]^{N-1} \sum_{1 \leqq j<k \leqq N} \int_{0}^{2 \pi} d \sigma_{j} \int_{0}^{2 \pi} d \sigma_{k} \hat{I}_{\mu_{1} \ldots \check{\mu}_{J} \ldots \check{\mu}_{k} \ldots \mu_{N}}^{ \pm c l}\left(\sigma_{j}, \sigma_{k}\right) \\
& \times\left[\frac{\delta}{i \delta x_{\mu_{j}}\left(\sigma_{j}\right)} \hat{u}_{\mu_{k}}^{ \pm}\left(\sigma_{k}\right)+(j \leftrightarrow k)\right]
\end{aligned}
$$

where the operator $\left(\frac{\delta}{i \delta x_{\mu_{j}}\left(\sigma_{j}\right)}\right)_{\mathrm{WKB}}$ acts only on the next to leading order in the $1 / M^{2}$-expansion of the wave-functional $\phi(\mathscr{C})$ of the state to which $\mathscr{Z}_{\mu_{1} \ldots \mu_{N}}^{ \pm}$has been applied. The symbol $\hat{R}_{\mu_{1} \ldots \mu_{n}}^{ \pm c l}(\sigma, \lambda), \sigma \geqq \lambda$ denotes

$$
\hat{R}_{\mu_{1} \ldots \mu_{n}}^{ \pm c l}(\sigma, \lambda)=\int_{\lambda}^{\sigma} d \sigma_{1} \int_{\lambda}^{\sigma_{1}} d \sigma_{2} \ldots \int_{\lambda}^{\sigma_{n}-1} d \sigma_{n} \hat{u}_{\mu_{1}}^{ \pm}\left(\sigma_{1}\right) \ldots \hat{u}_{\mu_{n}}^{ \pm}\left(\sigma_{n}\right)=\hat{R}_{\mu_{1} \ldots \mu_{n}}^{ \pm c l}(\sigma+2 \pi, \lambda+2 \pi)
$$

The operators $\hat{u}_{\mu}^{ \pm}(\sigma)$ act "classically":

$$
\begin{gathered}
\hat{R}_{\mu_{1} \ldots \mu_{n}}^{ \pm c l}(\sigma, \lambda) \phi(\mathscr{C})=R_{\mu_{1} \ldots \mu_{n}}^{ \pm}(\sigma, \lambda) \phi(\mathscr{C}), \\
R_{\mu_{1} \ldots \mu_{n}}^{ \pm}(\sigma, \lambda) \doteq \int_{\lambda}^{\sigma} d \sigma_{1} \int_{\lambda}^{\sigma_{1}} d \sigma_{2} \ldots \int_{\lambda}^{\sigma_{n}-1} d \sigma_{n} u_{\mu_{1}}^{ \pm}\left(\sigma_{1}\right) \ldots u_{\mu_{n}}^{ \pm}\left(\sigma_{n}\right), \\
u_{\mu}^{ \pm}(\sigma) \doteq i p_{\mu}(\sigma) \pm x_{\mu}^{\prime}(\sigma),
\end{gathered}
$$

where $p_{\mu}(\sigma)$ stands for the factor in front of the wave-functional $\phi(\mathscr{C})$ resulting from the classical action of the operator $\hat{p}_{\mu}(\sigma) \doteq-\frac{\delta}{M^{2} \delta x_{\mu}(\sigma)} \cdot p_{\mu}$ will be assumed to be a continuous function of the curve parameter $\sigma$.

Finally:

$$
\begin{aligned}
& \hat{I}_{\mu_{1} \ldots \breve{\mu}_{j} \ldots \check{\mu}_{k} \ldots \mu_{N}}^{ \pm c l}\left(\sigma_{j}, \sigma_{k}\right) \doteq \theta\left(\sigma_{j}-\sigma_{k}\right) \hat{R}_{\mu_{k+1} \ldots \mu_{N} \ldots \mu_{j-1}}^{ \pm c l}\left(2 \pi+\sigma_{k}, \sigma_{j}\right) \cdot \hat{R}_{\mu_{j+1} \ldots \mu_{k-1}}^{ \pm c l}\left(\sigma_{j}, \sigma_{k}\right) \\
& \quad+\theta\left(\sigma_{k}-\sigma_{j}\right) \hat{R}_{\mu_{k+1} \ldots \mu_{N} \ldots \mu_{j-1}}^{ \pm c l}\left(\sigma_{k}, \sigma_{j}\right) \cdot \hat{R}_{\mu_{j+1} \ldots \mu_{k-1}}^{ \pm c l}\left(2 \pi+\sigma_{j}, \sigma_{k}\right) .
\end{aligned}
$$


The symbol ... $\check{a} \ldots$ indicates the omission of the object $a$. Temporarily, we shall now identify the operators $\hat{u}_{\mu}^{ \pm}(\sigma), \hat{R}^{ \pm c l} \ldots(\sigma, \lambda)$, and $\hat{I}^{ \pm c l} \ldots\left(\sigma_{j}, \sigma_{k}\right)$ with the factors $u_{\mu}(\sigma), R^{ \pm} \ldots(\sigma, \lambda)$, and $I^{ \pm} \ldots\left(\sigma_{j}, \sigma_{k}\right)$ they produce when acting on the wavefunctional $\phi(\mathscr{C})$.

Notice that the "probing" functions $R_{\mu_{j+1} \ldots \mu_{N} \ldots \mu_{j-1}}^{ \pm}\left(2 \pi+\sigma_{j}, \sigma_{j}\right)$ and $I_{\mu_{1} \ldots \breve{\mu}_{J} \ldots \breve{\mu}_{k} \ldots \mu_{N}}^{ \pm}\left(\sigma_{j}, \sigma_{k}\right)$ are periodic of period $2 \pi$ in each one of their arguments: in $\sigma_{j}$ and in $\sigma_{j}$ and $\sigma_{k}$, respectively. Only the contribution

$$
\begin{aligned}
& {\left[M^{2}\right]^{N-1} / 2 \sum_{1 \leqq j<k \leqq N} \int_{0}^{2 \pi} d \sigma_{j} \int_{0}^{2 \pi} d \sigma_{k} I_{\mu_{1} \ldots \check{\mu}_{j} \ldots \breve{\mu}_{k} \ldots \mu_{N}}^{ \pm}\left(\sigma_{j}, \sigma_{k}\right)} \\
& \quad \times\left[\frac{\delta}{\delta x_{\mu_{j}}\left(\sigma_{j}\right)}\left\{p_{\mu_{k}}\left(\sigma_{k}\right) \mp i x_{\mu_{k}}^{\prime}\left(\sigma_{k}\right)\right\}+(j \leftrightarrow k)\right]
\end{aligned}
$$

requires definition. The latter one is achieved with the help of the short distance expansion [2]:

$$
\begin{aligned}
\frac{\delta}{\delta x_{\mu}(\sigma)} p_{v}(\lambda)= & -\frac{\left|x^{\prime}(\sigma)\right| \cdot\left|x^{\prime}(\lambda)\right|}{\pi(\Delta s)^{2}}\left\{\delta_{\mu \nu}-\frac{x_{\mu}^{\prime} x_{v}^{\prime}+p_{\mu} p_{v}}{x^{\prime 2}}\right\}(\sigma) \\
& -\frac{1}{\pi(\Delta s)}\left\{\left(\frac{x_{\mu}^{\prime}}{\left|x^{\prime}\right|}\right)^{\prime} x_{v}^{\prime}+\left(\frac{p_{\mu}}{\left|x^{\prime}\right|}\right)^{\prime} p_{v}\right. \\
& \left.+\left(\frac{x^{\prime \prime} \cdot p}{\left|x^{\prime}\right|^{3}}\right) x_{[\mu}^{\prime} p_{v]}\right\}(\sigma)+\mathcal{O}(\ln |\Delta s|),
\end{aligned}
$$

where $\Delta s$ is the shorter arc length between $x(\sigma)$ and $x(\lambda)$ and where along the shorter arc, with our convention on the parametrization of the curve $\mathscr{C}, \sigma$ is larger than $\lambda$.

We concentrate on the infinite subtractions which are needed in WKBapproximation. For that we rewrite

$$
\begin{aligned}
& {\left[M^{2}\right]^{N-1} / 2 \int_{0}^{2 \pi} d \sigma_{j} \int_{0}^{2 \pi} d \sigma_{k} I_{\mu_{1} \ldots \check{\mu}_{j} \ldots \check{\mu}_{k} \ldots \mu_{N}}^{ \pm}\left(\sigma_{j}, \sigma_{k}\right)} \\
& \quad \times\left[\frac{\delta}{\delta x_{\mu_{j}}\left(\sigma_{j}\right)}\left\{p_{\mu_{k}}\left(\sigma_{k}\right) \mp i x_{\mu_{k}}^{\prime}\left(\sigma_{k}\right)\right\}+(j \leftrightarrow k)\right] \\
& =\left[M^{2}\right]^{N-1} / 4 \int_{0}^{2 \pi} d \sigma_{j} \int_{0}^{2 \pi} d \sigma_{k}\left\{I_{\mu_{1}}^{ \pm} \ldots \check{\mu}_{j} \ldots \check{\mu}_{k} \ldots \mu_{N}\left(\sigma_{j}, \sigma_{k}\right)\right. \\
& \left.\quad-I_{\mu_{1} \ldots \breve{\mu}_{j} \ldots \breve{\mu}_{k} \ldots \mu_{N}}^{ \pm}\left(\sigma_{k}, \sigma_{j}\right)\right\} \frac{\delta p_{\left.\mu_{k}\right]}\left(\sigma_{k}\right)}{\delta x_{\left[\mu_{j}\right.}\left(\sigma_{j}\right)} \\
& +\left[M^{2}\right]^{N-1} / 4 \int_{0}^{2 \pi} d \sigma_{j} \int_{0}^{2 \pi} d \sigma_{k}\left\{I_{\mu_{1} \ldots \check{\mu}_{j} \ldots \breve{\mu}_{k} \ldots \mu_{N}}^{ \pm}\left(\sigma_{j}, \sigma_{k}\right)\right. \\
& \left.\quad+I_{\mu_{1} \ldots \breve{\mu}_{j} \ldots \check{\mu}_{k} \ldots \mu_{N}}^{ \pm}\left(\sigma_{k}, \sigma_{j}\right)\right\} \\
& \quad \times\left[\frac{\delta}{\delta x_{\mu_{j}}\left(\sigma_{j}\right)}\left\{p_{\mu_{k}}\left(\sigma_{k}\right) \mp i x_{\mu_{k}}^{\prime}\left(\sigma_{k}\right)\right\}+(j \leftrightarrow k)\right] .
\end{aligned}
$$


It is the second term which requires special attention:

$$
\begin{aligned}
& I_{\mu_{1} \ldots \breve{\mu}_{j} \ldots \breve{\mu}_{k} \ldots \mu_{N}}^{ \pm}\left(\sigma_{j}, \sigma_{k}\right)+I_{\mu_{1} \ldots \breve{\mu}_{j} \ldots \breve{\mu}_{k} \ldots \mu_{N}}^{ \pm}\left(\sigma_{k}, \sigma_{j}\right) \\
&= \theta\left(\sigma_{j}-\sigma_{k}\right)\left[R_{\mu_{k+1} \ldots \mu_{N} \ldots \mu_{J-1}}^{ \pm}\left(2 \pi+\sigma_{k}, \sigma_{j}\right) R_{\mu_{j}+1 \ldots \mu_{k-1}}^{ \pm}\left(\sigma_{j}, \sigma_{k}\right)\right. \\
&\left.+R_{\mu_{k+1} \ldots \mu_{N} \ldots \mu_{j-1}}^{ \pm}\left(\sigma_{j}, \sigma_{k}\right) R_{\mu_{j+1} \ldots \mu_{k-1}}^{ \pm}\left(2 \pi+\sigma_{k}, \sigma_{j}\right)\right] \\
&+\theta\left(\sigma_{k}-\sigma_{j}\right)\left[R_{\mu_{k+1} \ldots \mu_{N} \ldots \mu_{j-1}}^{ \pm}\left(2 \pi+\sigma_{j}, \sigma_{k}\right) R_{\mu_{j+1} \ldots \mu_{k-1}}^{ \pm}\left(\sigma_{k}, \sigma_{j}\right)\right. \\
&\left.+R_{\mu_{k+1} \ldots \mu_{N} \ldots \mu_{j-1}}^{ \pm}\left(\sigma_{k}, \sigma_{j}\right) R_{\mu_{j+1} \ldots \mu_{k-1}}^{ \pm}\left(2 \pi+\sigma_{j}, \sigma_{k}\right)\right] .
\end{aligned}
$$

This is a periodic function $(1 \leqq j<k \leqq N)$ which is in the general case

a) continuously differentiable at least with a double zero at $\sigma_{j}=\sigma_{k}$ provided $k-j \geqq 3$ and $N-k+j \geqq 3$;

b) continuous with a simple zero and a discontinuous first derivative at $\sigma_{j}=\sigma_{k}$ provided $k-j=2$ and $N-k+j \geqq 2$ or $N-k+j=2$ and $k-j \geqq 2$;

c) continuous with the finite value:

$$
R_{\mu_{k+1} \ldots \mu_{N} \ldots \mu_{j-1}}^{ \pm}\left(2 \pi+\sigma_{j}, \sigma_{j}\right)\left(R_{\mu_{2} \ldots \mu_{N-1}}^{ \pm}\left(2 \pi+\sigma_{j}, \sigma_{j}\right)\right)
$$

and a discontinuous first derivative at $\sigma_{j}=\sigma_{k}$ for $k=j+1(j=1$ and $k=N)$.

Case a) does not require subtractions. Case c) can be reduced to case b):

$$
\begin{aligned}
& {\left[M^{2}\right]^{N-1} / 4 \sum_{j=1}^{N} \int_{0}^{2 \pi} d \sigma_{j} \int_{0}^{2 \pi} d \sigma_{j+1}} \\
& \times\left\{I_{\mu_{1} \ldots \check{\mu}_{j} \check{\mu}_{j+1} \ldots \mu_{N}}^{ \pm}\left(\sigma_{j}, \sigma_{j+1}\right)+I_{\mu_{1} \ldots \check{\mu}_{j} \check{\mu}_{J+1} \ldots \mu_{N}}^{ \pm}\left(\sigma_{j+1}, \sigma_{j}\right)\right\} \\
& \times\left[\frac{\delta}{\delta x_{\mu_{j}}\left(\sigma_{j}\right)}\left\{p_{\mu_{j+1}}\left(\sigma_{j+1}\right) \mp i x_{\mu_{j+1}}^{\prime}\left(\sigma_{j+1}\right)\right\}+(j \leftrightarrow j+1)\right] \\
& =-\left[M^{2}\right]^{N-2} / 4 \sum_{j=1}^{N} \int_{0}^{2 \pi} d \sigma R_{\mu_{j+2} \mu_{j+3} \ldots \mu_{j-1}}^{ \pm}(2 \pi+\sigma, \sigma) \\
& \times\left[\frac{\delta}{\delta x_{\mu_{\mathrm{J}}}(\sigma)} P_{\mu_{\mathrm{J}+1}}^{c l}+\frac{\delta}{\delta x_{\mu_{\mathrm{J}+1}}(\sigma)} P_{\mu_{j}}^{c l}\right] \\
& +\left[M^{2}\right]^{N-1} / 4 \sum_{j=1}^{N} \int_{0}^{2 \pi} d \sigma_{j} \int_{0}^{2 \pi} d \sigma_{j+1}\left\{\theta ( \sigma _ { j } - \sigma _ { j + 1 } ) \left[R_{\mu_{j+2} \mu_{j+3} \ldots \mu_{j-1}}^{ \pm}\left(2 \pi+\sigma_{j+1}, \sigma_{j}\right)\right.\right. \\
& \left.-R_{\mu_{j+2} \mu_{J+3} \ldots \mu_{J-1}}^{ \pm}\left(2 \pi+\sigma_{j}, \sigma_{j}\right)+R_{\mu_{j+2} \mu_{j+3} \ldots \mu_{j-1}}^{ \pm}\left(\sigma_{j}, \sigma_{j+1}\right)\right] \\
& +\theta\left(\sigma_{j+1}-\sigma_{j}\right)\left[R_{\mu_{j+2} \mu_{j+3} \ldots \mu_{j-1}}^{ \pm}\left(2 \pi+\sigma_{j}, \sigma_{j+1}\right)\right. \\
& \left.\left.-R_{\mu_{j+2} \mu_{j+3} \ldots \mu_{j-1}}^{ \pm}\left(2 \pi+\sigma_{j}, \sigma_{j}\right)+R_{\mu_{j+2} \mu_{j+3} \ldots \mu_{j-1}}^{ \pm}\left(\sigma_{j+1}, \sigma_{j}\right)\right]\right\} \\
& \times \frac{\delta}{\delta x_{\mu_{j}}\left(\sigma_{j}\right)}\left\{p_{\mu_{j+1}}\left(\sigma_{j+1}\right) \mp i x_{\mu_{j+1}}^{\prime}\left(\sigma_{j+1}\right)\right\} \\
& +\left[M^{2}\right]^{N-1} / 4 \sum_{j=1}^{N} \int_{0}^{2 \pi} d \sigma_{j+1} \int_{0}^{2 \pi} d \sigma_{j}\left\{\theta ( \sigma _ { j + 1 } - \sigma _ { j } ) \left[R_{\mu_{j+2} \mu_{j+3} \ldots \mu_{j-1}}^{ \pm}\left(2 \pi+\sigma_{j}, \sigma_{j+1}\right)\right.\right. \\
& \left.-R_{\mu_{j+2} \mu_{j+3} \ldots \mu_{j-1}}^{ \pm}\left(2 \pi+\sigma_{j+1}, \sigma_{j+1}\right)+R_{\mu_{j+2} \mu_{j+3} \ldots \mu_{j-1}}^{ \pm}\left(\sigma_{j+1}, \sigma_{j}\right)\right]+\theta\left(\sigma_{j}-\sigma_{j+1}\right) \\
& \times\left[R_{\mu_{j+2} \mu_{j+3} \ldots \mu_{j-1}}^{ \pm}\left(2 \pi+\sigma_{j+1}, \sigma_{j}\right)-R_{\mu_{j+2} \mu_{j+3} \ldots \mu_{j-1}}^{ \pm}\left(2 \pi+\sigma_{j+1}, \sigma_{j+1}\right)\right. \\
& \left.\left.+R_{\mu_{j+2} \mu_{j+3} \ldots \mu_{j-1}}^{ \pm}\left(\sigma_{j}, \sigma_{j+1}\right)\right]\right\} \frac{\delta}{\delta x_{\mu_{j+1}}\left(\sigma_{j+1}\right)}\left\{p_{\mu_{j}}\left(\sigma_{j}\right) \mp i x_{\mu_{j}}^{\prime}\left(\sigma_{j}\right)\right\} \text {. }
\end{aligned}
$$

We have employed an enumeration modulo $N$. 
In order to proceed, we replace the remaining step functions by their regularized expressions

$$
\theta_{\delta}\left(\sigma_{1}, \sigma_{2}\right)= \begin{cases}1 & \text { if } \int_{\sigma_{2}}^{\sigma_{1}} d \sigma\left|x^{\prime}(\sigma)\right| \geqq \delta>0 \\ 0 & \text { otherwise }\end{cases}
$$

and determine the divergence of the correspondingly regularized expression for $\mathscr{Z}_{\mu_{1} \ldots \mu_{N}}^{ \pm}$as the invariant cut-off parameter $\delta$ tends to zero. (The singularities at the endpoints of the various integrations are integrable!) For $N \geqq 3$, the regularized expression for $\mathscr{Z}_{\mu_{1} \ldots \mu_{N}}^{ \pm}$diverges like

$$
\begin{aligned}
& \frac{\left[M^{2}\right]^{N-1}}{2 \pi} \ln \delta \sum_{j=1}^{N} \int_{0}^{2 \pi} d \sigma\left\{\left(x^{\prime 2}\right)^{-1}\left[x_{\left[\mu_{j}\right.}^{\prime \prime} x_{\left.\mu_{j+1}\right]}^{\prime}+\hat{p}_{\left[\mu_{j}\right.}^{\prime} \hat{p}_{\left.\mu_{J+1}\right]}+2\left(\frac{x^{\prime \prime} \cdot \hat{p}}{x^{\prime 2}}\right) x_{\left[\mu_{j}\right.}^{\prime} \hat{p}_{\left.\mu_{j+1}\right]}\right](\sigma)\right. \\
& \quad \times \hat{R}_{\mu_{j}+2 \mu_{j}+3 \ldots \mu_{j-1}}^{ \pm c l}(2 \pi+\sigma, \sigma)-\left[\delta_{\mu_{j} \mu_{j+1}}-\left(x^{\prime 2}\right)^{-1}\left(x_{\mu_{j}}^{\prime} x_{\mu_{j+1}}^{\prime}+\hat{p}_{\mu_{j}} \hat{p}_{\mu_{j+1}}\right)\right](\sigma) \\
& \quad \times\left[\hat{u}_{\mu_{j+2}}^{ \pm}(\sigma) \hat{R}_{\mu_{J+3} \ldots c l}^{ \pm \ldots \mu_{j-1}}(2 \pi+\sigma, \sigma)+\hat{R}_{\mu_{j+2} \ldots \mu_{j-2}}^{ \pm c l}(2 \pi+\sigma, \sigma) \hat{u}_{\mu_{j-1}}^{ \pm}(\sigma)\right] \\
& \quad+2\left[\delta_{\mu_{j} \mu_{j+2}}-\left(x^{\prime 2}\right)^{-1}\left(x_{\mu_{j}}^{\prime} x_{\mu_{j+2}}^{\prime}+\hat{p}_{\mu_{j}} \hat{p}_{\mu_{j+2}}\right)\right](\sigma) \\
& \left.\quad \times\left[\hat{R}_{\mu_{j+3} \ldots \mu_{j-1}}^{ \pm c l}(2 \pi+\sigma, \sigma) \hat{u}_{\mu_{j+1}}^{ \pm}(\sigma)+\delta_{N 4} \hat{P}_{\mu_{j+1}}^{c l} \hat{u}_{\mu_{j-1}}^{ \pm}(\sigma)\right]\right\} .
\end{aligned}
$$

For $N=3$ the last two terms inside the curled bracket must be omitted.

After having added corresponding counterterms to the regularized expression for $\mathscr{Z}_{\mu_{1} \ldots \mu_{N}}^{ \pm}$and subsequently having removed the regularization, we obtain a bona fide definition for $\mathscr{Z}_{\mu_{1} \ldots \mu_{N}}^{ \pm}$in WKB-approximation. At first sight, the subtractions seem to be attended with new free parameters. Actually, this point is rather subtle because of possible admixtures of total derivatives. For an analogous problem discussed further below there appears just one (already familiar) parameter!

Now we are in a position to show that the state described by the wave-functional $\psi(\mathscr{C})$ is left invariant under the action of the infinitesimal generators $\mathscr{Z}_{\mu_{1} \ldots \mu_{N}}^{ \pm}$of symmetry transformations - at least in WKB-approximation.

i) In the first section we have already established the invariance in the classical approximation: $\mathscr{Z}_{\mu_{1} \ldots \mu_{N}}^{ \pm c l} \psi(\mathscr{C})=0$.

ii) For the wave-functional $\psi(\mathscr{C})$ :

$$
R_{\mu_{1} \ldots \mu_{n}}^{ \pm}(\sigma, \lambda)=R_{\mu_{1} \ldots \mu_{n}}^{ \pm \psi}(z(\sigma), z(\lambda)), \quad n=1,2, \ldots
$$

Hence

$$
R_{\mu_{1} \ldots \mu_{n}}^{ \pm}(2 \pi+\sigma, \sigma)=0, \quad n=1,2, \ldots
$$

and consequently

$$
\left[M^{2}\right]^{N-1} \sum_{j=1}^{N} \oint d \sigma_{j} R_{\mu_{j+1} \mu_{j+2} \ldots \mu_{j-1}}^{ \pm}\left(2 \pi+\sigma_{j}, \sigma_{j}\right)\left(\frac{\delta}{i \delta x_{\mu_{j}}\left(\sigma_{j}\right)}\right)_{\mathrm{WKB}} \psi(\mathscr{C})=0
$$


iii) For the same reason, the remaining terms of $\mathscr{Z}_{\mu_{1} \ldots \mu_{N}}^{ \pm}$when acting on $\psi(\mathscr{C})$ do not require any subtractions. Their effect is uniquely determined:

$$
\begin{aligned}
& \frac{\left[M^{2}\right]^{N-1}}{2} \sum_{1 \leqq j<k \leqq N} \int_{0}^{2 \pi} d \sigma_{j} \int_{0}^{2 \pi} d \sigma_{k} I_{\mu_{1} \ldots \breve{\mu}_{j} \ldots \breve{\mu}_{k} \ldots \mu_{N}}^{ \pm}\left(\sigma_{j}, \sigma_{k}\right) \\
& \quad \times\left[\frac{\delta}{\delta x_{\mu_{j}}\left(\sigma_{j}\right)} u_{\mu_{k}}\left(\sigma_{k}\right)+(j \leftrightarrow k)\right] \psi(\mathscr{C}) \\
& =\frac{\left[\mathrm{M}^{2}\right]^{N-1}}{2} \sum_{j=1}^{N} \int_{0}^{2 \pi} d \sigma_{j}\left[\frac{\delta}{\delta x_{\mu_{j}}\left(\sigma_{j}\right)} R_{\mu_{j+1} \mu_{j+2} \ldots \mu_{j-1}}^{ \pm}\left(2 \pi+\sigma_{j}, \sigma_{j}\right)\right] \psi(\mathscr{C})=0 .
\end{aligned}
$$

Summarizing, we have proved in WKB-approximation that the state with wave-functional $\psi(\mathscr{C})$ is annihilated by every one of the invariant charges $\mathscr{Z}_{\mu_{1}}^{ \pm} \ldots \mu_{N}$, i.e. that this particular state is invariant under the transformations generated by the charges $\mathscr{Z}_{\mu_{1} \ldots \mu_{N}}^{ \pm}, \mu_{i}=1, \ldots, d, i=1, \ldots, N, N=(1,2) 3,4, \ldots$.

This result could have been anticipated by the following consideration. According to the analysis of [1], apart from the case $N=1: \mathscr{Z}_{\mu}^{ \pm}=P_{\mu}$, classically each invariant charge $\mathscr{Z}_{\mu_{1} \ldots \mu_{N}}^{ \pm}$is a polynomial of second or higher degree in the building blocks

$$
\begin{aligned}
\hat{R}_{v_{1} \ldots v_{r}}^{ \pm}= & \hat{R}_{v_{1} \ldots v_{r}}^{ \pm}(0) \\
& \doteq\left[M^{2}\right]^{r} \int_{0}^{2 \pi} d \sigma_{1} \ldots \int_{0}^{2 \pi} d \sigma_{r} \theta\left(\sigma_{1}-\sigma_{2}\right) \ldots \theta\left(\sigma_{r-1}-\sigma_{r}\right) \hat{u}_{v_{1}}^{ \pm}\left(\sigma_{1}\right) \ldots \hat{u}_{v_{r}}^{ \pm}\left(\sigma_{r}\right)
\end{aligned}
$$

with the classical restriction on the action of the operators $\hat{u}_{v_{j}}^{ \pm}\left(\sigma_{j}\right)$. If in the WKBapproximation to the quantum theory the effect of the charge $\mathscr{Z}_{\mu_{1} \ldots \mu_{N}}^{ \pm}$on the state with wave-functional $\psi(\mathscr{C})$ can still be viewed as being produced by operating with the same polynomial in the corresponding suitably renormalized building blocks $\hat{R}_{v_{1} \ldots v_{r}}^{ \pm}$on $\psi(\mathscr{C})$ - the order of the factors in each monomial being prescribed somehow - then $\mathscr{Z}_{\mu_{1} \ldots \mu_{N}}^{ \pm}$annihilates this state. The reason is that for each monomial at least one "classical" factor $R^{ \pm \psi} \ldots(z(2 \pi), z(0))$ is being produced possibly as a whole acted upon by some operator $\hat{u}_{v_{j}}^{ \pm}\left(\sigma_{j}\right)$ from some other building block - and this "classical" factor vanishes as has been shown before.

Indeed, there are two very appealing alternatives to define the action of the building blocks $\hat{R}_{\mu_{1} \ldots \mu_{N}}^{ \pm}$on $\psi(\mathscr{C})$ in WKB-approximation. The first alternative, again denoted by $\hat{R}_{\mu_{1} \ldots \mu_{N}}^{ \pm}$, corresponds to naive path-ordering the second one, denoted by $\hat{R}_{\mu_{1} \ldots \mu_{N}}^{ \pm}$, to naive anti path-ordering of the operators $\hat{u}_{\mu_{j}}^{ \pm}\left(\sigma_{j}\right)$. Formally:

$$
\begin{aligned}
\hat{R}_{\mu_{1} \ldots \mu_{N}}^{ \pm}= & \int_{0}^{2 \pi} d \sigma_{1} \ldots \int_{0}^{2 \pi} d \sigma_{N} \theta\left(\sigma_{1}-\sigma_{2}\right) \ldots \theta\left(\sigma_{N-1}-\sigma_{N}\right) \\
& \times\left[\frac{\delta}{i \delta x_{\mu_{1}}\left(\sigma_{1}\right)} \pm M^{2} x_{\mu_{1}}^{\prime}\left(\sigma_{1}\right)\right] \ldots\left[\frac{\delta}{i \delta x_{\mu_{N}}\left(\sigma_{N}\right)} \pm M^{2} x_{\mu_{N}}^{\prime}\left(\sigma_{N}\right)\right], \\
\overline{\hat{R}}_{\mu_{1} \ldots \mu_{N}}^{ \pm}= & \int_{0}^{2 \pi} d \sigma_{1} \ldots \int_{0}^{2 \pi} d \sigma_{N} \theta\left(\sigma_{1}-\sigma_{2}\right) \ldots \theta\left(\sigma_{N-1}-\sigma_{N}\right) \\
& \times\left[\frac{\delta}{i \delta x_{\mu_{N}}\left(\sigma_{N}\right)} \pm M^{2} x_{\mu_{N}}^{\prime}\left(\sigma_{N}\right)\right] \ldots\left[\frac{\delta}{i \delta x_{\mu_{1}}\left(\sigma_{1}\right)} \pm M^{2} x_{\mu_{1}}^{\prime}\left(\sigma_{1}\right)\right] .
\end{aligned}
$$


In order to determine the necessary subtractions for $\hat{R}_{\mu_{1} \ldots \mu_{N}}^{ \pm}$and $\bar{R}_{\mu_{1} \ldots \mu_{N}}^{ \pm}$ respectively, we proceed - to begin with - in close analogy to ordinary perturbation theory. There time-ordering and anti time-ordering of Wick ordered products of free field operators have to be defined. We observe that according to the formula

$$
\begin{aligned}
& \theta\left(\sigma_{1}-\sigma_{2}\right) \ldots \theta\left(\sigma_{N-1}-\sigma_{N}\right)+(-1)^{N} \theta\left(\sigma_{N}-\sigma_{N-1}\right) \ldots \theta\left(\sigma_{2}-\sigma_{1}\right) \\
& \quad=-\sum_{n=1}^{N-1}(-1)^{n} \theta\left(\sigma_{n}-\sigma_{n-1}\right) \ldots \theta\left(\sigma_{2}-\sigma_{1}\right) \cdot \theta\left(\sigma_{n+1}-\sigma_{n+2}\right) \ldots \theta\left(\sigma_{N-1}-\sigma_{N}\right)
\end{aligned}
$$

the action of the sum

$$
\hat{R}_{\mu_{1} \ldots \mu_{N}}^{ \pm}+(-1)^{N} \overline{\hat{R}}_{\mu_{N} \ldots \mu_{1}}^{ \pm}
$$

on $\psi(\mathscr{C})$ is already defined (in WKB-approximation) when the actions of all $\hat{R}_{\mu_{1} \ldots \mu_{n}}^{ \pm}$ and $\hat{R}_{\mu_{1} \ldots \mu_{n}}^{ \pm}$with $n<N$ on $\psi(\mathscr{C})$ have been defined (in WKB-approximation):

$$
\hat{R}_{\mu_{1} \ldots \mu_{N}}^{ \pm}+(-1)^{N} \overline{\hat{R}}_{\mu_{N} \ldots \mu_{1}}^{ \pm}
$$

annihilates $\psi(\mathscr{C})$. Thus it suffices to define the action of

$$
\hat{R}_{\mu_{1} \ldots \mu_{N}}^{ \pm}-(-1)^{N} \overline{\hat{R}}_{\mu_{N} \ldots \mu_{1}}
$$

on $\psi(\mathscr{C})$ in WKB-approximation.

The definition is achieved with the help of the short distance expansion [2] quoted above.

Replacing the step functions $\theta$ by $\theta_{\delta}$, we obtain the regularized expression

with

$$
\left[M^{2}\right]^{N} A_{\mu_{1} \ldots \mu_{N}}^{\delta}+\left[M^{2}\right]^{N-1}\left(B_{\mu_{1} \ldots \mu_{N}}^{\delta}+C_{\mu_{1} \ldots \mu_{N}}^{\delta}\right)
$$

$$
\begin{aligned}
& A_{\mu_{1} \ldots \mu_{N}}^{\delta} \doteq \int_{0}^{2 \pi} d \sigma_{1} \ldots \int_{0}^{2 \pi} d \sigma_{N} \theta_{\delta}\left(\sigma_{1}, \sigma_{2}\right) \ldots \theta_{\delta}\left(\sigma_{N-1}, \sigma_{N}\right) \\
& \times \chi_{\mu_{1}}^{ \pm \prime}\left(z\left(\sigma_{1}\right)\right) \ldots \chi_{\mu_{N}}^{ \pm \prime}\left(z\left(\sigma_{N}\right)\right)-(-1)^{N}\left(\begin{array}{c}
\mu_{1} \ldots \mu_{N} \\
\mu_{N} \ldots \mu_{1}
\end{array}\right), \\
& B_{\mu_{1} \ldots \mu_{N}}^{\delta} \doteq \int_{0}^{2 \pi} d \sigma_{1} \ldots \int_{0}^{2 \pi} d \sigma_{N} \theta_{\delta}\left(\sigma_{1}, \sigma_{2}\right) \ldots \theta_{\delta}\left(\sigma_{N-1}, \sigma_{N}\right) \\
& \times \sum_{n=1}^{N} \chi_{\mu_{1}}^{ \pm \prime}\left(z\left(\sigma_{1}\right)\right) \ldots \chi_{\mu_{n-1}}^{ \pm \prime}\left(z\left(\sigma_{n-1}\right)\right) \\
& \times\left[\frac{1}{i} \frac{\delta}{\delta x_{\mu_{n}}\left(\sigma_{n}\right)}\left(\gamma \kappa(\mathscr{C})-\frac{1}{2} W_{P V}\left(\Delta^{T}\right)\right)\right] \\
& \times \chi_{\mu_{n+1}}^{ \pm \prime}\left(z\left(\sigma_{n+1}\right)\right) \ldots \chi_{\mu_{N}}^{ \pm \prime}\left(z\left(\sigma_{N}\right)\right)-(-1)^{N}\left(\begin{array}{c}
\mu_{1} \ldots \mu_{N} \\
\mu_{N} \ldots \mu_{1}
\end{array}\right) \text {, } \\
& C_{\mu_{1} \ldots \mu_{N}}^{\delta} \doteq \int_{0}^{2 \pi} d \sigma_{1} \ldots \int_{0}^{2 \pi} d \sigma_{N} \theta_{\delta}\left(\sigma_{1}, \sigma_{2}\right) \ldots \theta_{\delta}\left(\sigma_{N-1}, \sigma_{N}\right) \\
& \left.\times \sum_{i \leqq j<k \leqq N} \chi_{\mu_{1}}^{ \pm \prime}\left(z\left(\sigma_{1}\right)\right) \ldots \overline{\chi_{\mu_{j}}^{ \pm \prime}\left(z\left(\sigma_{j}\right)\right.}\right) \overline{\ldots \chi_{\mu_{k}}^{ \pm \prime}\left(z\left(\sigma_{k}\right)\right)} \ldots \chi_{\mu_{N}}^{ \pm \prime}\left(z\left(\sigma_{N}\right)\right) \\
& \times \frac{\delta p_{\mu_{k}}\left(\sigma_{k}\right)}{\delta x_{\mu_{j}}\left(\sigma_{j}\right)}-(-1)^{N}\left(\begin{array}{l}
\mu_{1} \ldots \mu_{N} \\
\mu_{N} \ldots \mu_{1}
\end{array}\right) .
\end{aligned}
$$


As before, the symbol ... $\check{a}$... indicates the omission of the object $a$. As the cut-off $\delta$ tends to zero from above, $A_{\mu_{1} \ldots \mu_{N}}^{\delta}$ tends to

$$
R_{\mu_{1} \ldots \mu_{N}}^{ \pm \psi}(z(2 \pi), z(0))-(-1)^{N} R_{\mu_{N} \ldots \mu_{1}}^{ \pm \psi}(z(2 \pi), z(0))=0,
$$

$B_{\mu_{1} \ldots \mu_{N}}^{\delta}$ tends to

$$
\begin{aligned}
& \int_{0}^{2 \pi} d \sigma_{1} \ldots \int_{0}^{2 \pi} d \sigma_{N} \sum_{n=1}^{N} \chi_{\mu_{1}}^{ \pm \prime}\left(z\left(\sigma_{1}\right)\right) \ldots \chi_{\mu_{n}}^{ \pm \prime}\left(z\left(\sigma_{n}\right)\right) \ldots \chi_{\mu_{N}}^{ \pm \prime}\left(z\left(\sigma_{N}\right)\right) \\
& \times\left[\frac{1}{i} \frac{\delta}{\delta x_{\mu_{n}}\left(\sigma_{n}\right)}\left(\gamma \kappa(\mathscr{C})-\frac{1}{2} W_{P V}\left(\Delta^{T}\right)\right)\right]-(-1)^{N}\left(\begin{array}{l}
\mu_{1} \ldots \mu_{N} \\
\mu_{N} \ldots \mu_{1}
\end{array}\right)
\end{aligned}
$$

and $C_{\mu_{1} \ldots \mu_{N}}^{\delta}$ diverges logarithmically like

$$
\begin{gathered}
\frac{|\ln \delta|}{\pi} \oint d s\left\{\sum _ { n = 1 } ^ { N - 1 } ( - 1 ) ^ { n - 1 } \left[R_{\mu_{n-1} \ldots \mu_{1}}^{ \pm \psi} e_{\mu_{n}}^{i}\left(D_{s} e_{\mu_{n+1}} R_{\mu_{n+2} \ldots \mu_{N}}^{ \pm \psi}\right)^{i}\right.\right. \\
\left.-\left(D_{s} R_{\mu_{n-1} \ldots \mu_{1}}^{ \pm \psi} e_{\mu_{n}}\right)^{i} e_{\mu_{n+1}}^{i} R_{\mu_{n+2} \ldots \mu_{N}}^{ \pm \psi}\right] \\
\left.-2 \sum_{n=2}^{N-1}(-1)^{n-2} R_{\mu_{n-2} \ldots \mu_{1}}^{ \pm \psi} e_{\mu_{n-1}}^{i} \dot{\chi}_{\mu_{n}}^{ \pm} e_{\mu_{n+1}}^{i} R_{\mu_{n+2} \ldots \mu_{N}}^{ \pm \psi}\right\}
\end{gathered}
$$

with

$$
R_{v_{1} \ldots v_{r}}^{ \pm \psi}=R_{v_{1} \ldots v_{r}}^{ \pm \psi}(z(\sigma), z(0))
$$

Here $D_{s}$ denotes the tangential covariant derivative $D_{s} \doteq \dot{z}^{a} D_{a}$. Similarly, in the following $D_{n}$ will denote the normal covariant derivative $D_{n} \doteq n^{a} D_{a}$.

The diverging part of $C_{\mu_{1} \ldots \mu_{N}}^{\delta}$ can be reexpressed

$$
\begin{aligned}
& \frac{|\ln \delta|}{\pi}\left\{\sum_{n=1}^{N-1}(-1)^{n-1} \oint d s R_{\mu_{n-1} \ldots \mu_{1}}^{ \pm \psi} e_{\left[\mu_{n}\right.}^{i}\left(D_{s} e\right)_{\left.\mu_{n+1}\right]}^{i} R_{\mu_{n+2} \ldots \mu_{N}}^{ \pm \psi}\right. \\
& \quad \pm i \sum_{n=2}^{N-1}(-1)^{n-2} \int_{\Gamma} d^{2} z|g|^{1 / 2} g^{a b} R_{\mu_{n-2} \ldots \mu_{1}}^{ \pm \psi}\left[\left(D_{a} e\right)_{\left[\mu_{n-1}\right.}^{i} e_{\left.\mu_{n}\right]}^{i}\left(\partial_{b} \chi_{\mu_{n+1}}^{ \pm}\right)\right. \\
& \left.\left.\quad-\left(\partial_{a} \chi_{\mu_{n-1}}^{ \pm}\right)\left(D_{b} e\right)_{\left[\mu_{n}\right.}^{i} e_{\left.\mu_{n+1}\right]}^{i}\right] R_{\mu_{n+2} \ldots \mu_{N}}^{ \pm \psi}\right\} .
\end{aligned}
$$

Here we have used Gauss' theorem, the differential equation for minimal surfaces and the relations

$$
\left(\partial_{a} \chi_{\mu}^{ \pm}\right) g^{a b}\left(\partial_{b} \chi_{\nu}^{ \pm}\right)=0, \quad\left(D_{a} e\right)_{[\mu}^{i} g^{a b}\left(\partial_{b} \chi_{v]}^{ \pm}\right)=0 .
$$

The last relation is proved again with the help of the differential equation of minimal surfaces, here in the form

$$
g^{a b} V_{a b}^{i}=0, \quad i=1,2, \ldots, d-2 .
$$

We claim: $\left[M^{2}\right]^{N-1}$ times the diverging part of $C_{\mu_{1} \ldots \mu_{N}}^{\delta}$ is cancelled by the factor which is produced when the following counter term - to be added to the regularized expression for $\hat{R}_{\mu_{1} \ldots \mu_{N}}^{ \pm}-(-1)^{N} \hat{R}_{\mu_{N} \ldots \mu_{1}}^{ \pm}-$is applied to the state with 
wave-functional $\psi(\mathscr{C})$. (The operators

act "classically"!)

$$
\left[\frac{1}{i} \frac{\delta}{\delta x_{\mu_{j}}\left(\sigma_{j}\right)} \pm i M^{2} x_{\mu_{j}}^{\prime}\left(\sigma_{j}\right)\right]
$$

$$
\begin{aligned}
- & \frac{|\ln \delta|}{4 \pi} \int_{0}^{2 \pi} d \sigma_{1} \ldots \int_{0}^{2 \pi} d \sigma_{N}\left[\theta\left(\sigma_{1}-\sigma_{2}\right) \ldots \theta\left(\sigma_{N-1}-\sigma_{N}\right)\right. \\
& \left.-(-1)^{N} \theta\left(\sigma_{N}-\sigma_{N-1}\right) \ldots \theta\left(\sigma_{2}-\sigma_{1}\right)\right] \\
& \times \sum_{n=1}^{N}\left[\frac{1}{i} \frac{\delta}{\delta x_{\mu_{1}}\left(\sigma_{1}\right)} \pm M^{2} x_{\mu_{1}}^{\prime}\left(\sigma_{1}\right)\right] \ldots\left[\frac{1}{i} \frac{\delta}{\delta x_{\mu_{n}}\left(\sigma_{n-1}\right)} \pm M^{2} x_{\mu_{n-1}}^{\prime}\left(\sigma_{n-1}\right)\right] \frac{\delta \kappa(\mathscr{C})}{i \delta x_{\mu_{n}}\left(\sigma_{n}\right)} \\
& \times\left[\frac{1}{i} \frac{\delta}{\delta x_{\mu_{n+1}}\left(\sigma_{n+1}\right)} \pm M^{2} x_{\mu_{n+1}}^{\prime}\left(\sigma_{n+1}\right)\right] \ldots\left[\frac{1}{i} \frac{\delta}{\delta x_{\mu_{N}}\left(\sigma_{N}\right)} \pm M^{2} x_{\mu_{N}}^{\prime}\left(\sigma_{N}\right)\right] .
\end{aligned}
$$

If this is true, then the subtraction may be considered as being generated essentially by the functional

$$
\exp \{-\Delta S\}, \quad \Delta S=\frac{|\ln \delta|}{2 \pi} \int_{\Gamma} d^{2} z|g|^{1 / 2} K=\frac{|\ln \delta|}{4 \pi} \kappa(\mathscr{C})
$$

and the only free parameter coincides with the parameter $\gamma$.

We shall now proceed to verify the above claim. To this end we apply the debatable subtraction operator to $\psi(\mathscr{C})$. The wave-functional $\psi(\mathscr{C})$ is reproduced up to the factor

$$
\begin{aligned}
& -\left[M^{2}\right]^{N-1} \frac{|\ln \delta|}{4 \pi i} \sum_{n=1}^{N}(-1)^{n-1} \int_{0}^{2 \pi} d \sigma R_{\mu_{n-1} \ldots \mu_{1}}^{ \pm \psi}(z(\sigma), z(0)) \frac{\delta \kappa(\mathscr{C})}{\delta x_{\mu_{n}}(\sigma)} \\
& \times R_{\mu_{n+1} \ldots \mu_{N}}^{ \pm \psi}(z(\sigma), z(0))-(-1)^{N}\left(\begin{array}{l}
\mu_{1} \ldots \mu_{N} \\
\mu_{N} \ldots \mu_{1}
\end{array}\right) .
\end{aligned}
$$

Using the Gauss-Bonnet formula,

we obtain

$$
\kappa(\mathscr{C})=2 \int_{\Gamma} d^{2} z|g|^{1 / 2} K=4 \pi+2 \int_{0}^{2 \pi} d \lambda(p \cdot \ddot{x})(\lambda),
$$

$$
\begin{aligned}
\frac{\delta \kappa(\mathscr{C})}{\delta x_{\mu}(\sigma)}= & 2\left|x^{\prime}(\sigma)\right|\left\{K(z(\sigma)) \frac{p_{\mu}(\sigma)}{\left|x^{\prime}(\sigma)\right|}\right. \\
& \left.+e_{\mu}^{i}(z(\sigma))\left[D_{s}^{i j}\left(\left(\frac{p}{\left|x^{\prime}\right|}\right) e^{j}\right)(\sigma)-\left(D_{n} h\right)^{i}(z(\sigma))\right]\right\},
\end{aligned}
$$

where the quantities $h^{i}(z), i=1, \ldots, d-2$ are defined by the following conditions:

$$
(H h)^{i}(z)=0 \quad \text { for } \quad z \in \stackrel{\circ}{\Gamma}, \quad h^{i}(z(\sigma))=\left(\dot{x}(\sigma) \cdot\left(D_{s} e\right)^{i}(z(\sigma))\right) .
$$

When we insert this expression for the functional derivative $\frac{\delta \kappa(\mathscr{C})}{\delta x_{\mu}(\sigma)}$, integrate by
parts and use the following identity:

$$
|K(z(\sigma))| \frac{p_{\mu}(\sigma)}{\left|x^{\prime}(\sigma)\right|}=\left(\frac{p}{\left|x^{\prime}\right|} \cdot\left(D_{s} e\right)^{i}\right)(\sigma)\left(D_{s} e\right)_{\mu}^{i}(z(\sigma))+\left(\frac{p}{\left|x^{\prime}\right|} \cdot\left(D_{n} e\right)^{i}\right)(\sigma)\left(D_{n} e\right)_{\mu}^{i}(z(\sigma)),
$$


we can bring the factor in question into the form

$$
\begin{aligned}
& {\left[M^{2}\right]^{N-1} \frac{|\ln \delta|}{2 \pi i} \sum_{n=1}^{N}(-1)^{n-1} \oint d s} \\
& \times\left\{R _ { \mu _ { n - 1 } \ldots \mu _ { 1 } } ^ { \pm \psi } ( z ( s ) , z ( 0 ) ) \left[\left(\frac{p}{\left|x^{\prime}\right|} \cdot\left(D_{n} e\right)^{i}\right)(s)\left(D_{n} e\right)_{\mu_{n}}^{i}(z(s))\right.\right. \\
& \left.+e_{\mu_{n}}^{i}(z(s))\left(D_{n} h\right)^{i}(z(s))\right] R_{\mu_{n+1} \ldots \mu_{N}}^{ \pm}(z(s), z(0)) \\
& -R_{\mu_{n-2} \ldots \mu_{1}}^{ \pm \psi}(z(s), z(0)) \dot{\chi}_{\mu_{n-1}}^{ \pm}(z(s))\left(\frac{p}{\left|x^{\prime}\right|} \cdot\left(D_{s} e\right)^{i}\right)(s) \\
& \times e_{\mu_{n}}^{i}(z(s)) R_{\mu_{n+1} \ldots \mu_{N}}^{ \pm \psi}(z(s), z(0)) \\
& -R_{\mu_{n-1} \ldots \mu_{1}}^{ \pm \psi}(z(s), z(0))\left(\frac{p}{\left|x^{\prime}\right|} \cdot\left(D_{s} e\right)^{i}\right)(s) e_{\mu_{n}}^{i}(z(s)) \\
& \left.\times \dot{\chi}_{\mu_{n+1}}^{ \pm}(z(s)) R_{\mu_{n+2} \ldots \mu_{N}}^{ \pm \psi}(z(s), z(0))\right\}-(-1)^{N}\left(\begin{array}{c}
\mu_{1} \ldots \mu_{N} \\
\mu_{N} \ldots \mu_{1}
\end{array}\right) \text {. }
\end{aligned}
$$

Further, using the fact that $\left(D_{s} e\right)_{\mu}^{i}(z(s))$ and $\left(D_{n} e\right)_{\mu}^{i}(z(s))$ are vectors in the linear span of $\left(\frac{p_{\mu}}{\left|x^{\prime}\right|}\right)(s)$ and $\dot{x}_{\mu}(s)$ and taking advantage of the following relations:

$$
\left(\frac{p}{\left|x^{\prime}\right|} \cdot\left(D_{s} e\right)^{i}\right)(s)=\left(\dot{x} \cdot\left(D_{n} e\right)^{i}\right)(s), \quad\left(\frac{p}{\left|x^{\prime}\right|} \cdot\left(D_{n} e\right)^{i}\right)(s)=-\left(\dot{x} \cdot\left(D_{s} e\right)^{i}\right)(s)
$$

- the latter one is derived from the differential equation of minimal surfaces in the form $g^{a b} V_{a b}^{i}=0, i=1,2, \ldots, d-2$ - we arrive at

$$
\begin{aligned}
& -\left[M^{2}\right]^{N-1} \frac{|\ln \delta|}{\pi}\left\{\sum _ { n = 1 } ^ { N - 1 } ( - 1 ) ^ { n - 1 } \oint d s \left[R_{\mu_{n-1} \ldots \mu_{1}}^{ \pm \psi} e_{\left[\mu_{n}\right.}^{i}\left(D_{s} e\right)_{\left.\mu_{n+1}\right]}^{i} R_{\mu_{n}+2 \ldots \mu_{N}}^{ \pm \psi}\right.\right. \\
& \left.+i R_{\mu_{n-1} \ldots \mu_{1}}^{ \pm \psi}\left(D_{n} e\right)_{\left[\mu_{n}\right.}^{i} e_{\left.\mu_{n+1}\right]}^{i} R_{\mu_{n+2} \ldots \mu_{N}}^{ \pm \psi}\right] \\
& +\frac{1}{i} \sum_{n=1}^{N}(-1)^{n-1} \oint d s\left[h^{i}\left(D_{n} R_{\mu_{n}-1 \ldots \mu_{1}}^{ \pm \psi} e_{\mu_{n}} R_{\mu_{n}+1 \ldots \mu_{N}}^{ \pm \psi}\right)^{i}\right. \\
& \left.\left.-R_{\mu_{n-1} \ldots \mu_{1}}^{ \pm \psi} e_{\mu_{n}}^{i} R_{\mu_{n+1} \ldots \mu_{N}}^{ \pm \psi}\left(D_{n} h\right)^{i}\right]\right\} .
\end{aligned}
$$

Finally, by applying Gauss' theorem, the relation

$$
\left(\partial_{a} \chi_{\mu}^{ \pm}\right) g^{a b}\left(\partial_{b} \chi_{v}^{ \pm}\right)=0
$$

and the differential equations

$$
(H e)_{\mu}^{i}=0, \quad(H h)^{i}=0, \quad-|g|^{-1 / 2} \partial_{a}|g|^{1 / 2} g^{a b} \partial_{b} \chi_{\mu}^{ \pm}=0,
$$


we are led to

$$
\begin{aligned}
- & {\left[M^{2}\right]^{N-1} \frac{|\ln \delta|}{\pi}\left\{\sum_{n=1}^{N-1}(-1)^{n-1} \oint d s R_{\mu_{n-1} \ldots \mu_{1}}^{ \pm \psi} e_{\left[\mu_{n}\right.}^{i}\left(D_{s} e\right)_{\left.\mu_{n+1}\right]}^{i} R_{\mu_{n}+2 \ldots \mu_{N}}^{ \pm \psi}\right.} \\
& \pm i \sum_{n=2}^{N-1}(-1)^{n-2} \int_{\Gamma} d^{2} z|g|^{1 / 2} g^{a b} R_{\mu_{n-2} \ldots \mu_{1}}^{ \pm \psi}\left[\left(D_{a} e\right)_{\left[\mu_{n-1}\right.}^{i} e_{\left.\mu_{n}\right]}^{i}\left(\partial_{b} \chi_{\mu_{n+1}}^{ \pm}\right)\right. \\
& \left.\left.-\left(\partial_{a} \chi_{\mu_{n-1}}^{ \pm}\right)\left(D_{b} e\right)_{\left[\mu_{n}\right.}^{i} e_{\left.\mu_{n+1}\right]}^{i}\right] R_{\mu_{n+2} \ldots \mu_{N}}^{ \pm \psi}\right\} \\
& +2 i\left[M^{2}\right]^{N-1} \frac{|\ln \delta|^{N-1}}{\pi} \sum_{n=1}^{N-1}(-1)^{n-1} \int_{\Gamma} d^{2} z|g|^{1 / 2} g^{a b} h^{i} R_{\mu_{n-1} \ldots \mu_{1}}^{ \pm \psi} \\
& \times\left(D_{a} e\right)_{\left[\mu_{n}\right.}^{i}\left(\partial_{b} \chi_{\left.\mu_{n+1}\right]}^{ \pm}\right) R_{\mu_{n+2} \ldots \mu_{N}}^{ \pm \psi} .
\end{aligned}
$$

The last sum vanishes on account of the relation

$$
\left(D_{a} e\right)_{[\mu}^{i} g^{a b}\left(\partial_{b} \chi_{v]}^{ \pm}\right)=0 .
$$

Thus we are left with exactly the right subtraction to cancel the divergence of the regularized expression for $\hat{R}_{\mu_{1} \ldots \mu_{N}}-(-1)^{N} \hat{R}_{\mu_{N} \ldots \mu_{1}}^{ \pm}$applied to $\psi(\mathscr{C})$ as the cut-off is removed. This completes the verification of the claim.

According to the above analysis, the necessary modifications of the action of the operators $\hat{R}_{\mu_{1} \ldots \mu_{N}}^{ \pm}$on the wave-functional $\psi(\mathscr{C})$ due to one loop order renormalization are well understood.

Actually, as to the structure of the counterterms, the operators $\hat{R}_{\mu_{1} \ldots \mu_{N}}^{ \pm}$are for the wave-functional $\psi(\mathscr{C})$ exactly what the invariant charges $\mathscr{Z}_{\mu_{1} \ldots \mu_{N}}^{ \pm}$are for the general string wave-functional $\phi(\mathscr{C})$. Hence, the renormalization of the charges $\mathscr{Z}_{\mu_{1} \ldots \mu_{N}}^{ \pm}$in WKB-approximation should be attended with a single free parameter related to $\gamma$. Moreover, it should be possible to fix this parameter in terms of $\gamma$ by requiring the renormalized invariant charges to commute with the renormalized loop wave-operator [2]. The latter one involves the parameter $\gamma$.

We shall return to these questions in a separate paper.

\section{References}

1. Pohlmeyer, K.: Lecture Notes in Physics, Vol. 226, 157. Berlin, Heidelberg, New York, Tokyo: Springer 1985

2. Lüscher, M., Symanzik, K., Weisz, P.: Anomalies of the free loop wave equation in the WKB approximation. Nucl. Phys. B 173, 365 (1980)

3. Nitsche, J.C.C.: Vorlesungen über Minimalflächen. Die Grundlehren der mathematischen Wissenschaften, Band 199. Berlin, Heidelberg, New York: Springer 1975

4. Pohlmeyer, K., Rehren, K.-H.: Algebraic properties of the invariant charges of the NambuGoto theory. Commun. Math. Phys. 105, 593-627 (1986)

Communicated by K. Osterwalder

Received January 27, 1986 
